\title{
Electromagnetic-Based Therapy
}

National Cancer Institute

\section{Source}

National Cancer Institute. Electromagnetic-Based Therapy. NCI Thesaurus. Code

C16207.

The unconventional use of electromagnetic fields for medical or therapeutic purposes. 